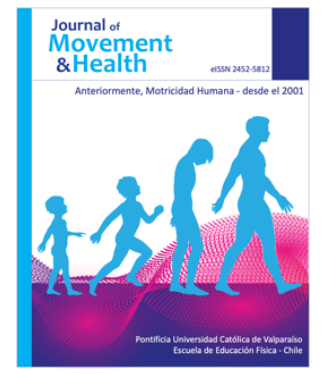

eISSN: 2452-5812

http://jmh.pucv.cl/

Recibido: $15 / 06 / 2021$

Aceptado: 28/06/2021

Disponible: $12 / 07 / 2021$

Publicado: 01/01/2022

\title{
Comentario
}

\section{Programas de acceso a pedagogías, ¿Una opción para estudiar Educación Física?}

Pedagogies access program, Is it an option to study Physical Education?

\author{
Villena, $\mathbf{N}^{1}$; Villegas, $A^{2}$; Castillo-Paredes, $A^{3,4}$ \\ Correspondencia \\ Dr. Antonio Castillo-Paredes \\ Escuela de Pedagogía en Educación Física, Facultad de Educación, Universidad de Las Américas, Santiago, \\ Chile. \\ acastillop85@gmail.com
}

\section{Puntos destacables}

- Se revisaron las vías acceso para las carreras de pedagogía determinadas por la Ley 20.903.

- Se examinaron las características particulares de los Programas de Acceso a Pedagogías reconocidos por el MINEDUC.

- Se sugiere considerar las características específicas de las carreras de Pedagogía en Educación Física en la construcción e implementación de los Programas de Acceso a Pedagogías para continuidad de estudios superiores.

En el marco de la Ley $20.129^{1}$ y la Ley $20.903^{2}$ que son reguladoras de procesos de acreditación de carreras y programas de pedagogía, las universidades en Chile están sujetas a que los postulantes a pedagogía cumplan con una serie de requisitos para estudiar estas carreras. Entre los criterios de acceso de la Ley $20.903^{2}$ encontramos 1) puntaje promedio de pruebas de acceso obligatorias, 2) ranking de Notas de Enseñanza Media (NEM), 3) combinación de puntaje pruebas de acceso y ranking NEM, y 4) Programas de Acceso a Pedagogías (PAP).

Los $\mathrm{PAP}^{3}$, constituyen una vía de acceso reconocida por la Ley 20.903 y cuentan con la aprobación del Ministerio de Educación (MINEDUC). Estos programas poseen características heterogéneas entre las diferentes universidades que los imparten, siendo aplicados a estudiantes de enseñanza media. A través de esta vía, los estudiantes de enseñanza media aseguran su continuidad de estudios superiores en carreras de pedagogía, para ello deben aprobar el programa PAP, y rendir la Prueba de Transición para la Admisión Universitaria $^{4}$, pero no se considera el puntaje obtenido en esta última.

Estos programas son transversales para aquellos estudiantes interesados en cursar una carrera de pedagogía, sin diferenciar componentes que reconozcan los diferentes perfiles de los estudiantes en función de la carrera que desean seguir. Esto también debería estar relacionado con un perfil base que debería existir a nivel nacional para las carreras de pedagogía, donde cada universidad, sobre esta base, complemente en función de las habilidades que requiere en los estudiantes para lograr su perfil de egreso.

Las carreras de pedagogías poseen características particulares que no están siendo consideradas, lo que nos invita a reflexionar sobre el concepto de una "educación de calidad". El aseguramiento de la calidad en educación superior se controla mediante la instauración de criterios básicos que garanticen el cumplimiento de los programas de estudio por parte de las instituciones y, además, mediante un proceso de evaluación de la calidad establecida a través de etapas de autoevaluación, evaluación externa y finalmente, el proceso de acreditación propiamente tal ${ }^{5}$. 
Un estudio elaborado el año 2020 por un grupo de académicos de universidades chilenas ${ }^{6}$, señala que la mayoría de las universidades ha creado programas que cumplen con el rol pedagógico, pero que existe diversidad en los propósitos, modelos de formación y mecanismos de acompañamiento, en donde sólo es posible ver dos grandes áreas en común como lo son el desarrollo de habilidades de escritura y matemática y lo relacionado a la vocación y rol docente.

De esta forma, los PAP se convierten en una vía de entrada, pero debiesen ser una oportunidad de ingreso temprano a la pedagogía que les permita a los estudiantes desarrollar una serie de habilidades iniciales para fortalecer su interés hacia las diferentes carreras de pedagogía. Este es el caso de la Pedagogía en Educación Física, la que debe dar respuesta a un contexto escolar enmarcado hacia la actividad física, ejercicio físico, deporte, salud, motricidad humana y, además, considerar aspectos pedagógicos tales como la sociedad, familia y cultura ${ }^{7,8}$.

En el caso de Chile, desde el MINEDUC, la asignatura "Educación Física y Salud” en contextos escolares declara que, mediante la práctica de actividad física de manera regular, el escolar debe desarrollar "habilidades motrices y actitudes proclives al juego limpio, el liderazgo y el autocuidado", además de considerar la "adquisición de un estilo de vida activo y saludable, orientado hacia el desarrollo de beneficios personales y sociales" ". Por otra parte, los estudiantes de pedagogía en Educación Física de Chile asocian la especialidad hacia la salud y el bienestar, integrando el movimiento y la motricidad humana, de esta forma asignan un valor pedagógico mediante elementos tales como la práctica de la actividad física, recreación y el deporte ${ }^{10}$.

De acuerdo con los antecedentes expuestos, la creación de estos programas especiales (PAP) buscan potenciar habilidades y disposiciones asociadas a la enseñanza de manera general, no obstante, estos programas no consideran las características particulares de cada carrera, como es el caso de la pedagogía en Educación Física, que en nuestros días debe dar respuesta a requerimientos gubernamentales, no gubernamentales y sociales.

\section{Referencias}

1. BNC. Ley 20129 Establece un Sistema Nacional de Aseguramiento de la Calidad de la Educación Superior. Accedido May 3, 2021. https://www.bcn.cl/leychile/navegar?idNorma=255323

2. BNC. Ley 20903 Crea el Sistema de Desarrollo Profesional Docente y Modifica Otras Normas. Accedido May 3, 2021. https://www.bcn.cl/leychile/navegar?idNorma=1087343

3. MINEDUC. PAP 2021. Programas de Acceso a Pedagogías. Accedido May 3, 2021. http://dfi.mineduc.cl/index2.php?id_seccion=5472\&id portal=59\&id contenido $=34645$

4. DEMRE. Temarios Pruebas de Admisión Transitorias a la Educación Superior 2021 - Admisión 2022. Accedido May 3, 2021. https://demre.cl/la-prueba/pruebas-y-temarios/presentacion-pruebastemarios-p2022

5. Zapata G, Clasing P. [El uso de criterios e indicadores de calidad en la acreditación de programas: diferencias entre agencias privadas de acreditación en Chile.]. Aseguramiento de la calidad en educación superior Chile. 2016; ISSN 0719-7896.

6. Hormazabal N, Abricot N, Oyarzo K, Alvarado M, Bravo, C. [Programas de acceso especial a las carreras de pedagogía: Sus características en las Universidades del Estado de Chile]. Sophia Austral. 2020; (25): 93-119. DOI: 10.4067/S0719-56052020000100093

7. Espejo R. Algunos aspectos de la educación compleja. Polis. Revista Latinoamericana. 2010; (25):119-135. DOI: 10.4067/S0718-65682010000100007

8. Santos M. El pensamiento complejo y la pedagogía: Bases para una teoría holística de la educación. Estudios pedagógicos (Valdivia). 2000; (26):133-148. DOI: 10.4067/S0718-07052000000100011 
DOI: https://doi.org/10.5027/jmh-Vol19-Issue1(2022)art132

9. MINEDUC. Educación Física y Salud. Accedido Jun 1, 2021. https://www.curriculumnacional.cl/portal/Educacion-General/Educacion-fisica-y-salud/

10. Caniuqueo A, Hernández-Mosqueira C, Troyano A, et al. Representaciones sociales: el significado de la Educación Física para los estudiantes de esa disciplina. Revista electrónica de investigación educativa. 2018; 20(1): 104-111. DOI: 10.24320/redie.2018.20.1.1497

Afiliaciones

${ }^{1}$ Escuela de Pedagogía en Educación Física, Facultad de Educación, Universidad de Las Américas, Viña del Mar, Chile.

${ }^{2}$ Escuela de Educación, Facultad de Educación, Universidad de Las Américas, Santiago, Chile.

${ }^{3}$ Grupo AFySE, Investigación en Actividad Física y Salud Escolar, Escuela de Pedagogía en Educación Física, Facultad de Educación, Universidad de Las Américas, Santiago, Chile.

${ }^{4}$ Grupo de Educación Física y Aprendizaje, Xalapa, Veracruz, México.

\section{Declaración de Autoría}

N.V; C.V; A.C.-P.: Acceso total a todos los datos del estudio y análisis de datos; diseño y redacción del manuscrito.

\section{Conflicto de interés}

Ninguno de los autores presentar conflicto de interés.

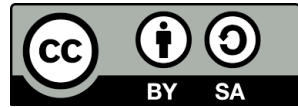

